\title{
Closed-loop laser polishing using in-process surface finish metrology
}

\author{
OLIVER FAEHNLE, ${ }^{1}$ ROLF RASCHER, ${ }^{2}$ CHRISTIAN VOGT ${ }^{2}$ AND DAE WOOK \\ $\mathrm{KIM}^{3, *}$
}

\author{
${ }^{1}$ FISBA AG, Rorschacher 268, CH-9016 St.Gallen, Switzerland \\ ${ }^{2}$ THD-Technische Hochschule Deggendorf, Germany \\ ${ }^{3}$ College of Optical Sciences, University of Arizona, Tucson, AZ 85721, USA \\ *Corresponding author: letter2dwk@hotmail.com
}

Received XX Month XXXX; revised XX Month, XXXX; accepted XX Month XXXX; posted XX Month XXXX (Doc. ID XXXXX); published XX Month XXXX

This paper lays out the trail onto a closed-loop polishing process of optical elements enabling the application of the optimum polishing time needed. To that aim, an in-process testing method for monitoring an inclusive micro surface quality (e.g., comprising surface roughness and scratch-and-dig) within the polishing spot is analyzed and its applicability to closed-loop polishing for classical loose-abrasive full-aperture polishing as well as for computer controlled laser polishing is experimentally tested and verified. This enables the determination of the optimum local dwell time resulting in a stable and cost optimized polishing. (C) 2017 Optical Society of America

OCIS codes: (220.0220) Optics design and fabrication; (220.4610) Optics fabrication; (220.5450) Polishing.

http://dx.doi.org/10.1364/AO.99.099999

Optical lenses have been generated for more than 3400 years, as indicated e.g. by the 1400 B.C. Minoan lenses which were excavated on the Greek Island of Crete [1]. If we analyze the development of optical fabrication technologies ever since and if we do this from an evolutionary point of view, we recognize that this process has been ruled by aiming on "minimum effort taken" to generate "just good enough levels of lens quality" needed; with lens quality essentially meaning shape accuracy and surface finish (comprising level of surface roughness (Sq), depth of subsurface damage (SSD) and level of scratch-and-dig (S\&D)).

\section{Introduction}

In the author's perception, we identify three sequential phases in the evolution of optical fabrication technology overlapping each other temporally: (a) handcrafting, (b) machining and (c) processing. These three phases distinguish themselves in their general strategy to generate the required lens quality level and put the significance of the presented closed-loop technology in context.

\section{A. Handcrafting (lens manufacturing without using machines)}

Our early forefathers have been rubbing blank materials such as crystal surfaces by handheld tools to generate shiny objects such as ritual gadgets, tools, weapons or jewelry for centuries. In doing so, the lens generation process (LGP) has been controlled by two entities: 1) cognitive thinking based on logic and 2) intuitive sensing using human's vision, audition and the sensation of hands.
This handcrafted LGP is even today still being applied by hobby astronomers polishing lenses without any access to machines.

B. Machining (lens production with kinematics carried out by machines guided by humans).

Step by step, parts of the LGP have been transferred to tooling and machining devices. For instance, during the development of abrasively grinding and polishing of lenses, the manual rotational movement of the lens was replaced by the use of bow-driven spindles. This type of machine resembles an early version of a lathe and has been in use for at least 1,940 years as described by Plinius 77 P.C. [2].

The combination of rotating the optical substrate (or the tool) by a machine and translating and pressing the tool (or the lens) by hand is still in existence today (e.g., foot-driven polishing machine in Figure 1). Master opticians are capable of hand polishing optical surfaces down to shape accuracies of below $30 \mathrm{~nm}$ RMS deviation of the desired shape and surface roughness levels of $\sim 0.5-2 \mathrm{~nm}$ RMS although they are using spindles featuring lateral positioning accuracies of several 100 microns.

This craftsmanship is often referred to as "the golden hands of the opticians", a skill one cannot learn by rational thinking only but by training your hand's sensation and intuition while feeling the LGP in situ. With machinery successively taking over the kinematics of handcrafting, we have been gaining accuracy and stability. Thus, major progresses have been made: e.g. feed controlled CNC machineries reaching positioning accuracies of less 
than $\sim 80 \mathrm{~nm}$ enabling a new process of ultra-precision machining: ductile mode grinding [3].
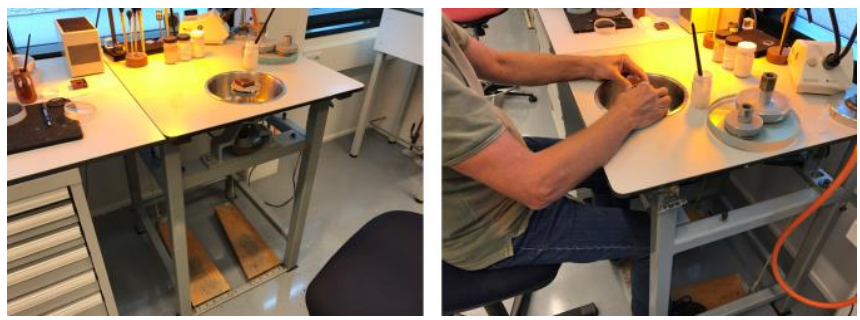

Fig. 1. Foot-driven polishing machine at an optics shop in Delft, The Netherlands.

\section{Processing (LGP optimized by controlling multiple fabrication} parameters without human interactions)

Modern optics manufacturing community has gained much progress by optimizing machines only. Nevertheless, we need to keep in mind that machines are only one part of the LGP and that on our trail to process stabilization and full automation in optics fabrication, we need to automate the whole lens generating process itself. To that aim, LGP quality improvement methods have been developed which strictly distinguish between machine and process issues identifying and optimizing critical fabrication process parameters and their interrelations [4].

The most recent stop along the "evolutionary" trail towards highly-efficient and fully-automated deterministic fabrication processes was the development of CCOS (Computer Controlled Optical Surfacing) technology. Here, the tool guidance on precision machines by cognitive thinking and intuition has been completely replaced by Computer Numerical Control machines and process optimization intelligence such as dwell-time/parameter simulation algorithms. Well known and industrially established CCOS platforms such as MRF (Magnetorheological Finishing) and IBF (Ion Beam Figuring) enable highest possible surface form qualities by using dwell-time controlled surfacing processes without depending on employees education (cognitive thinking) or optician's "golden hands".

\section{In-process Metrology for Closed-loop Polishing}

The surface finish can be measured after each iterative run using a portable phase shifting interferometer in situ (e.g., Micro Finish Topographer [5]) or a compact scattering-based roughness sensor (e.g., horos [6]) mounted on a machine or robot. These highly sensitive measurement technologies provide successfully accurate surface information to guide further iterative runs.

And yet, mainly due to the lack of in-process capability (i.e., simultaneous testing during fabrication) feedback mechanism, the process control during CCOS runs is done primarily as an openloop process (i.e., iterative approach of fabrication-measurementfabrication cycles without real-time feedback).

\section{A. Closed-loop polishing setup}

This paper reports on a new closed-loop approach for polishing processes by introducing an in-process (and in situ) monitoring of lens finish levels within a single compressed-but-inclusive signal as shown in Figure 2.

Besides the lens shaping process, polishing (the generation of the required level of surface finish) is the most expensive and time consuming process in many optics fabrication applications. Unfortunately, in order to set up polishing times, we are still mostly relying on human intuition and experience. Besides that, surface brightness is measured neither in situ nor in process but off-machine by measuring two parameters separately: Sq and S\&D.

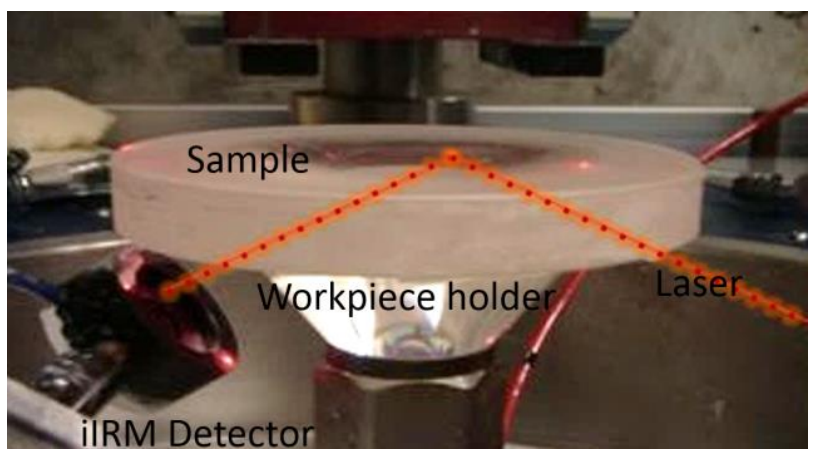

Fig. 2. iIRM: While the sample is being polished, the intensity of a laser beam is being detected that is internally reflected from within the material at the surface under test. That way in-process lens's surface quality is being monitored within a single signal.

In the presented approach, however, the optimum polishing time can be detected in real-time within the polishing spot enabling a cost and time optimization of the polishing process, getting full automation within reach. To that aim, the recently developed method for measuring surface quality, called intensitydetecting Internal Reflection Microscopy (iIRM) [7] shown in Figure 2, has been further developed and applied for in-process control of lens finish levels on machine while running traditional loose abrasive polishing process and a sub-aperture laser polishing process.

\section{B. ilRM metrology with sub-nanometer sensitivity}

It has been demonstrated that, by monitoring the intensity of a laser beam that is being reflected at an optical surface within the sample while its outside is being abrasively ground and polished, the surface finish levels can be monitored in-process [7].

Any surface imperfections such as Sq, S\&D or SSD cause scattering and consequently a loss of intensity of the reflected beam (i.e., Fresnel reflection) as an inclusive single output signal, which provides a simple-but-powerful closed-loop feedback system. It is important to note that it is not monitoring the scattered light distribution, which may include more information about the surface quality, but could be too sensitive to be implemented in the in-process polishing with the mechanical motions, vibrations and polishing slurry actions. Due to its simplicity measuring only the specular reflectance signal, the iIRM is robust enough to be applied during the actual manufacturing runs, which makes it a true in-process metrology method.

\section{Micro-roughness monitoring using ilRM}

An iIRM experimental study has been performed using two BK7 samples. The flat BK7 samples (1 inch diameter each) have been polished down to optical qualities using cerium oxide based slurries on a traditional spindle machine and their surface roughness values have been measured by whitelight interferometry as a reference. 
Both samples were mounted on the polishing machine spindle as shown in Figure 3 and the iIRM signal has been monitored (applying an angle of 5 degrees between the laser beam and the surface normal) while the setup is rotating. As the ilRM measures sample (a) and (b) alternatively, the recorded signal in Figure 3 clearly demonstrates distinguishing sub nanometer levels of surface roughness levels e.g. $0.6 \mathrm{~nm}$ rms from $1.1 \mathrm{~nm}$ rms.

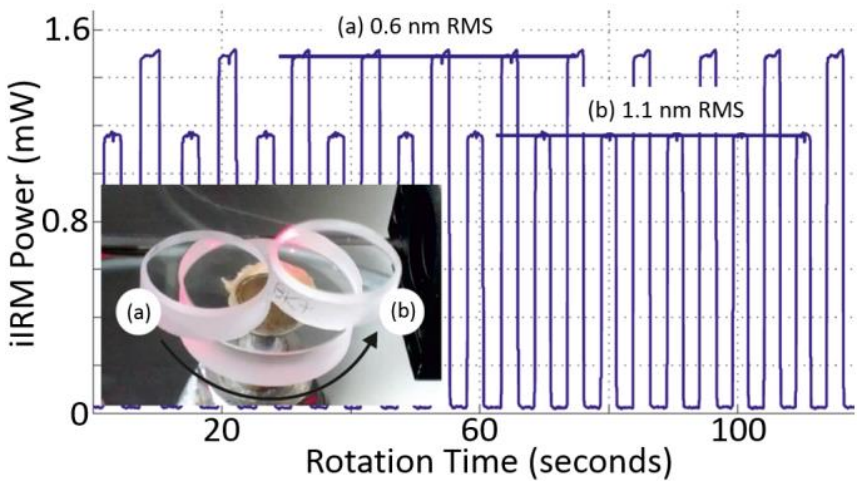

Fig. 3. Experimental iIRM measurements setup for sample (a) with Sq $=1.1 \mathrm{~nm}$ RMS and sample (b) with $\mathrm{Sq}=0.6 \mathrm{~nm}$ RMS. The measured iIRM power as a function of spindle rotating time is plotted in the graph. (Note: The reference (absolute) surface roughness values were measured using a whitelight interferometer.)

\section{Scratch-and-dig sensing using ilRM}

The iIRM's sensing capability to detect S\&D was experimentally verified by measuring three sample sleeves. The microscopic image of the sleeves are shown in Figure 4. A wide range of polishing defects from $900 \mathrm{~nm}$ deep brittle scratch to $100 \mathrm{~nm}$ sleeves without any brittle cracking present were detected as presented in Figure 4.

Consequently, iIRM is a comprehensive solution to achieve a closed-loop optics finishing by monitoring both surface finish levels (Sq and S\&D) using a single feedback control signal: in situ and in process.

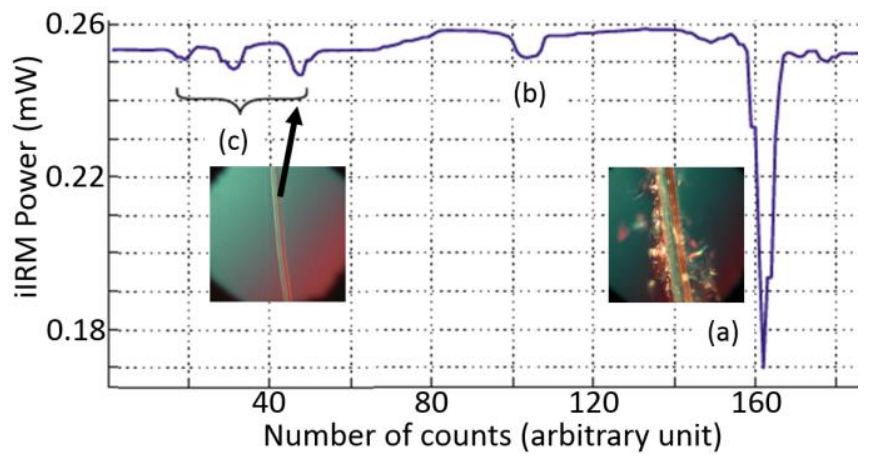

Fig. 4. iIRM measurement as it goes through generated sample grooves of (a) $900 \mathrm{~nm}$ deep brittle scratch, (b) $150 \mathrm{~nm}$ deep ductile sleeve and (c) three polishing sleeves - the smaller ones of which are about 100 $\mathrm{nm}$ deep. (Note: All groves were measured by whitelight interferometry as a reference to know the dimensions of S\&D's.)

\section{Closed-Loop Loose Abrasive Polishing}

\section{A. Real-time process monitoring}

The iIRM closed-loop concept was applied to a traditional LASF31 lens polishing process first. Figure 5 shows a typical iIRM signal characteristic which was measured on a traditional load controlled spindle polishing machine in situ and in process. As the lens surface finish level improves during polishing, less light is being scattered at the surface under test and the iIRM signal rises until the polishing process reaches (in this example after approximately 150 minutes) the ultimate level of lens surface quality achievable by the applied set of fabrication parameters. This is indicated by the iIRM signal reaching its final plateau. It is important to note that, after about half an hour of polishing, the polishing process got contaminated causing S\&D and a dip (red circle in Figure 5) in the signal which subsequently was polished away again. This highlights the real-time feedback loop capability of the ilRM based polishing technology.

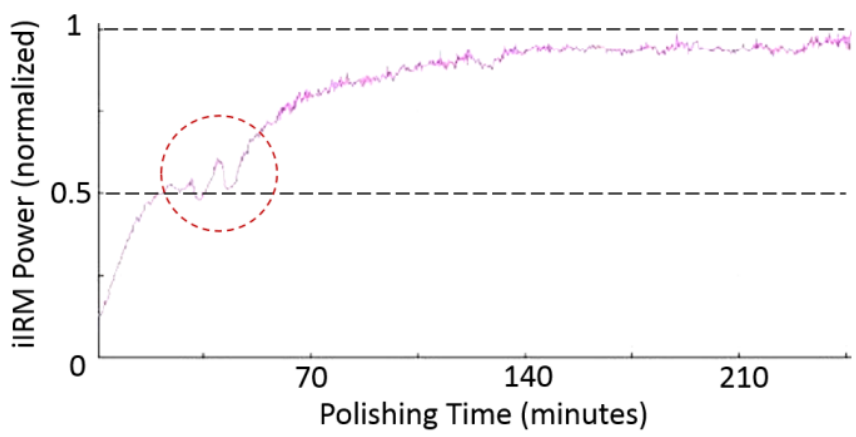

Fig. 5. iIRM: while the sample lens (LASF31) is being polished, the intensity of the reflected laser beam is being monitored: as lens finish level improves, less light is being scattered at the surface under test and the iIRM signal rises until the polishing process reaches after approximately 150 minutes the ultimate level of lens finish level achievable by the applied set of fabrication parameters.

Contrary to traditional methods, where setting up a polishing time is based on an empirical estimation (i.e., open-loop), employing iIRM in process, the exact moment is being detected at which the final plateau has been reached. From here on, polishing any longer is not improving lens finish level anymore and can be stopped for saving cost and machining time. Also, if necessary, the next manufacturing phase (e.g., IBF or MRF) can be started depending on the final target specification.

\section{B. Comparative experimental demonstration}

As a comparative demonstration of the iIRM's capability to be applied for closed-loop fabrication process control sensor, in Figure 6 two independent BK7 lens polishing processes were monitored and compared.

As predicted by Preston [8], for $4 \mathrm{~kg}$ load (i.e., higher polishing pressure) traditional polishing reaches its final level of lens finish quality earlier than if $1 \mathrm{~kg}$ load being applied. Therefore, monitoring the lens Fresnel reflection change applying iIRM, polishing can be stopped at the most efficient moment when the signal reaches its final plateau. 


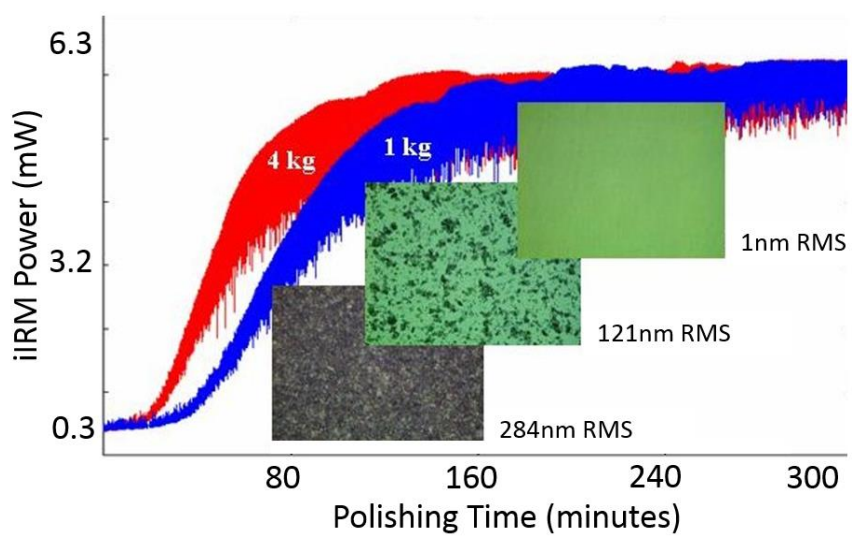

Fig. 6. Comparative closed-loop polishing processes for ground BK7 lens with different polishing loads of $1 \mathrm{~kg}$ and $4 \mathrm{~kg}$. (Note: Nomarski microscopy images of the surface in different phases of the process are inserted with their respective RMS surface roughness values.)

It is worth to note that one of the big limitations, especially in the industrial optics manufacturing process, has been the absence of in-process (i.e., real-time feedback during a polishing run) metrology sensing the creation/polishing/removal of S\&D. In order to check those surface quality status, the machine had to stop for a measurement or inspection. The measurement might be an in-situ approach, but it is still not a real-time. This often causes severe manufacturing efficiency and throughput issues. The same statement is applied for the real-time monitoring of surface roughness quality. As shown in Figure 6, using the in-process metrology feedback, a non-stop polishing run can be continued as a single run (i.e., closed-loop) until the surface quality meets the requirement without stopping (i.e., conventional "polish-stopmeasure-polish" open-loop) the machine.

\section{Closed-Loop $\mathrm{CO}_{2}$ Laser Polishing Technology}

Among the various sub-aperture polishing techniques of glass existing, laser polishing $[9,10]$, distinguishes itself by its scalable polishing spot size as well as by the absence of any physical contact between the tool and workpiece. Polishing is achieved by local absorption of laser power within the polishing spot (i.e., footprint) causing the surface and its subsurface region to melt and flow reducing surface roughness, defects and removing subsurface damage (SSD) as long as the necessary depth is molten.

To that aim, setting up process parameters is a tradeoff between continuous wave at lower laser power values and the application of a pulsed laser illumination at a high power level. Lower laser power is melting the surface and near surface regions gently but risking the generation of shape deviations and mid-spatial frequencies due to material flow. The application of a high power pulsed laser is avoiding changes in mid-spatial frequency surface shapes but risking high surface and subsurface tension caused by the high temperature gradients generated which might even cause local cracking. In both cases, the risk of vaporization of material exists which might cause re-deposition of material by the laser beam being scanned along the surface. Thus, in-process surface quality monitoring becomes a critical component to guide and optimize a laser polishing process.

\section{A. $\mathrm{CO}_{2}$ laser polishing experimental setup}

The ilRM method is applied for the determination and control of optimum local polishing dwell times, enabling a closed-loop laser polishing process called C-LasSO (Control of LASer Surface Optimization).

The experimental C-LasSO set-up to guide the laser polishing process of Fused Silica sample is shown in Figure 7. The ilRM HeNe (wavelength $\lambda=0.633 \mu \mathrm{m}$ ) laser is being reflected from the sample surface and the reflected beam intensity is being monitored. The internal local sub-aperture surface area under test is collocated at the exact laser polishing active area melting its surface locally. The closed-loop experimental configuration and set-up parameters are summarized in Table 1.

Table 1. Closed-Loop $\mathrm{CO}_{2}$ Laser Polishing Configuration

\begin{tabular}{|c|c|c|}
\hline & Specification & Extra Reference \\
\hline $\mathrm{CO}_{2}$ laser & $\begin{array}{l}120 \mathrm{~W} \text { at } \lambda=10.6 \mu \mathrm{m} \\
(2000 \mathrm{~W} \text { Max })\end{array}$ & $\begin{array}{l}\text { Coherent-ROFIN } \\
\mathrm{CO}_{2} \text { Slab laser }\end{array}$ \\
\hline $\begin{array}{l}\text { Polishing } \\
\text { coverage/speed }\end{array}$ & $\begin{array}{l}12 \times 12 \mathrm{~mm} \text { area } \\
\text { in } \sim 1 \text { minute }\end{array}$ & $\begin{array}{l}\text { E.g., } 2 \text { of } 15 \times 15 \mathrm{~mm} \\
\text { areas in Figure } 8\end{array}$ \\
\hline iIRM laser* & $\begin{array}{l}\text { HeNe laser } \\
\lambda=633 \mathrm{~nm}\end{array}$ & $\begin{array}{l}\text { or Diode laser } \\
2 \mathrm{~mW} \text { at } \lambda=650 \mathrm{~nm}\end{array}$ \\
\hline iIRM detector* & $\begin{array}{l}\text { Silicon Photodiode } \\
\lambda: 400-1100 \mathrm{~nm}\end{array}$ & $\begin{array}{l}\text { Thorlabs } \\
\text { PM16-120 }\end{array}$ \\
\hline Substrate Type & Fused Silica & $\begin{array}{l}\text { Fine ground } \\
\text { with 5-7 } \mu \mathrm{m} \mathrm{SiC} \mathrm{grit}\end{array}$ \\
\hline
\end{tabular}

* Note: This is a typical laser and detector configuration for a standard iIRM set-up. However, any other laser-detector pair can be used as long as it produces a repeatable and stable outcome signal as shown in Figure 3.

Since the fused silica is not transparent at the operating wavelength of the applied $\mathrm{CO}_{2}$ polishing laser (wavelength $\lambda=10.6$ $\mu \mathrm{m}$ ), the intensity of the reflected HeNe intensity can be monitored without any interfering signals. This unique C-LasSO configuration enables a real-time direct monitoring of the active laser polishing zone in process using a set of laser polishing parameters such as dwell time and applied laser power.

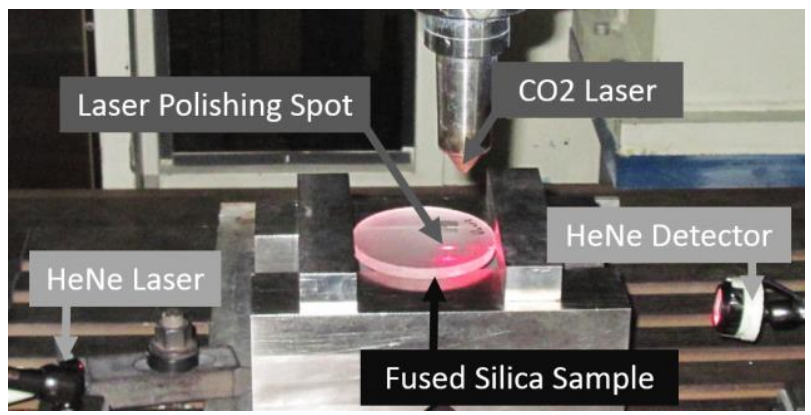

Fig. 7. C-LasSO set up for a closed-loop $\mathrm{CO}_{2}$ laser polishing process at the Technische Hochschule Deggendorf, Germany. While the Fused Silica sample is locally laser polished, the local surface roughness within the laser footprint is being monitored by detecting the intensity of a HeNe laser reflected right in the collocating $\mathrm{CO}_{2}$ laser footprint zone.

\section{B. Real-time $\mathrm{CO}_{2}$ laser polishing process monitoring}

Figure 8 (left) presents the history of measured iIRM signal monitoring the surface finish quality during the closed-loop process using C-LasSO. A fine ground fused silica sample $(\sim 166$ 
nm RMS surface roughness) was laser polished as shown in Figure 8 (right). As the surface roughness is improved due to the melting process, the C-LasSO signal increases until the final surface quality has been reached at about $2.7 \mathrm{~nm}$ RMS surface roughness and, the C-LasSO signal reaches a plateau. From that point on, the closedloop polishing process stops since any further laser polishing does not increase surface quality anymore but may worsen the surface quality.

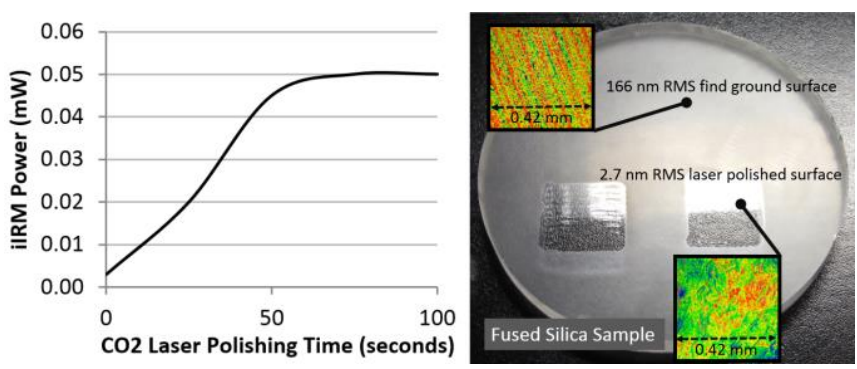

Fig. 8. (left) Measured iIRM signal in the C-LasSO set up. The signal was simultaneously recorded during the laser polishing process on a fine ground fused silica sample. (right) Two square regions were polished and both the initial ground surface and the final polished area were measured using a whitelight interferometer.

\section{Concluding Remarks}

The development in the field of optical fabrication technology throughout history has been analyzed from an evolutionary point of view identifying basically three sequential phases overlapping each other temporally: (a) handcrafting, (b) machining and (c) processing. While essential progress has been made optimizing machines only, enabling technologies such as ductile mode grinding, the next step is the optimization of fabrication processes themselves including machineries being one tessera within the whole process.

Following that trail, the process of monitoring optical surface finish during surface polishing has been proposed and analyzed. The first experimental results towards the closed-loop approach controlling the $\mathrm{CO}_{2}$ laser polishing process have been presented.

The iIRM, a compressed single Fresnel reflection measurement approach, has been adapted to monitor surface roughness and defect changes within the active polishing spot. This concept was experimentally verified by setting up a closed-loop polishing process. Based on the iIRM signal being monitored in process, the polishing run can be stopped at the optimal moment of meeting the surface finish specification to save machining time and cost.

The suitability of this closed-loop approach to both traditional loose abrasive polishing and laser polishing has been demonstrated and the experimental data have been reported. For traditional polishing, its capability of real-time detection of the very moment when no further surface finish improvement will occur was confirmed. In addition, by applying the C-LasSO method, it is possible to determine the optimal dwell time for a local laser polishing spot. Consequently, C-LasSO enables the optimization of laser polishing by avoiding excessive local dwell times and minimizing the risk of generating shape deviations such as mid-tohigh spatial frequency errors and a possible vaporization of glass. Also, this technology can be utilized for a laser polishing parameter set optimization by (e.g., $\mathrm{CO}_{2}$ laser power) monitoring the real-time surface quality evolution.
For the lens manufacturing case in Figure 2, both surfaces are polished and the iIRM laser beam was delivered through the bottom surface. If it is a mirror fabrication case, the same technique can be still applied since the back surface is often polished prior to the front (i.e., mirror) surface for mountingfixtures requiring stress-free flat back surface. However, thanks to the non-contact laser polishing process, C-LasSO (Patent Pending) is currently being tested in a mode sensing the polishing spot from the outside of the material instead of testing the internal reflection within the material. There is no more requirement for a polished bottom surface. Moreover, it will enable a development of an enhanced laser polishing process for strongly curved ground or polished optical surfaces.

\section{References}

1. G. Sines, and Y. Sakellarakis, "Lenses in Antiquity," American Journal of Archaeology 91(2), 191 (1987).

2. Plinius Secundus, "Naturalis Historia," 77 P.C.

3. T. G. Bifano, T. Dow, and R. Scattergood, “Ductile-Regime Grinding: a New Technology for Machining Brittle Materials," ASME Journal 113, 184 (1991).

4. O. W. Fähnle, "Process optimization in optical fabrication, " Opt. Eng. 55(3), 035106 (2016)

5. J. D. Hoyo, H. Choi, J. H. Burge, G. Kim, and D. W. Kim, “Experimental power spectral density analysis for mid- to high-spatial frequency surface error control," Appl. Opt. 56, 5258 (2017).

6. S. Schröder, A. von Finck, T. Herffurth, and A. Duparré, "Quality assessment of precision optical surfaces through light scattering techniques," in Imaging and Applied Optics Technical Papers, OSA Technical Digest 2012 (Optical Society of America, 2012), paper JTu5A.10.

7. O. Fähnle, Proc. SPIE 10326, "In process monitoring of optics fabrication," Fourth European Seminar on Precision Optics Manufacturing, 103260Y (2017).

8. F. W. Preston, "The theory and design of plate glass polishing machines," J. Soc. Glass Tech. 11: 214 (1927).

9. A. Richmann, E. Willenborg, and K. Wissenbach, "Laser Polishing of Fused Silica," in International Optical Design Conference and Optical Fabrication and Testing , OSA Technical Digest 2010 (Optical Society of America, 2010), paper OTuC2.

10. S. Heidrich, "Abtragprozesse and Prozesskette zur laserbasierten Fertigung optischer Elemente aus Quarzglas," Berichte aus der Lasertechnik, Shaker, Aachen (2014). 